\title{
Light Emission by Nanoporous GaN Produced by a Top-Down, Nonlithographical Nanopatterning
}

\author{
E. Kheirandish, ${ }^{1}$ N. A. Kouklin $\mathbb{D}^{1},{ }^{1}$ and J. Liang $\mathbb{D}^{2}$ \\ ${ }^{1}$ Department of Electrical Engineering and Computer Science, University of Wisconsin-Milwaukee, Milwaukee, WI, USA \\ ${ }^{2}$ Department of Mechanical Engineering, Worcester Polytechnic University, Worcester, MA, USA \\ Correspondence should be addressed to N. A. Kouklin; nkouklin@uwm.edu
}

Received 15 May 2018; Revised 27 August 2018; Accepted 4 September 2018; Published 4 October 2018

Academic Editor: Yasuhiko Hayashi

Copyright (C) 2018 E. Kheirandish et al. This is an open access article distributed under the Creative Commons Attribution License, which permits unrestricted use, distribution, and reproduction in any medium, provided the original work is properly cited.

\begin{abstract}
Temperature-dependent photoluminescence (PL) spectroscopy is carried out to probe radiative recombination and related light emission processes in two-dimensional periodic close-packed nanopore arrays in gallium nitride (np-GaN). The arrays were produced by nonlithographic nanopatterning of wurtzite GaN followed by a dry etching. The results of Raman spectroscopy point to a small relaxation of the compressive stress of $\sim 0.24 \mathrm{GPa}$ in nanoporous vs. bulk GaN. At $\sim 300 \mathrm{~K}$, the PL emission is induced by excitons and not free-carrier interband radiative recombinations. An evolution of the emission spectra with $\mathrm{T}$ is confirmed to be mainly a result of a decay of nonexcitonic PL emission and less of spectral shifts of the underlying PL bands. A switching of excitonic PL regime observed experimentally was analyzed within the exciton recombination-generation framework. The study provides new insights into the behaviors and physical mechanisms regulating light emission processes in $\mathrm{np}-\mathrm{GaN}$, critical to the development of nano-opto-electronic devices based on mesoscopic GaN.
\end{abstract}

\section{Introduction}

For its advanced electronic, optical, and physical characteristics including a direct band gap, excellent thermal conductivity, and electron mobility $>1400 \mathrm{~cm}^{2} / \mathrm{Vs}, \mathrm{GaN}$ remains one of the key, technologically advanced wide-band-gap semiconductors. [1-3]. The advent of electronic-grade p- and n-type GaN helped fuel the development of new solid-state light sources, photodetectors, high-power amplifiers, and radiation hard electronic devices [4-6]. Yet, while bulk GaN devices continue to be a focus of experimental and theoretical research, a significant effort has been recently devoted to producing crystalline nanoporous $\mathrm{GaN}$ (np-GaN) films and templates [7-9]. These mesostructures feature a number of profoundly different characteristics that can help propel applications of $\mathrm{GaN}$ beyond electronics. For instance, the refractive index of the np-GaN templates can be readily tuned to control light propagation/localization effects in their based solid-state devices by simply changing the pore size and density. In prior studies, the approach was used to achieve a manifold increase in the photon generation and extraction efficiencies in LEDs that implemented distributed Bragg reflectors based on $\mathrm{np}-\mathrm{GaN}[10,11]$. The use of nanoporous substrates was also shown to mitigate problems with heteroepitaxial growth of lattice-mismatched semiconductors and to enable synthesis of low-stress, electronic-grade GaN films and interfaces [12].

Today, several fabrication routes exist to produce $\mathrm{np}-\mathrm{GaN}$, with the most notable ones being electrochemical wet-etching, Au-based high-T surface decomposition, onestep annealing and laser ablation. Yet, the samples produced by these techniques typically suffer from broad diameter distributions, increased defect densities, and reduced crystal quality [13-15]. Alternatively, as we reported previously, highly ordered, high-density np-GaN can be readily produced by reactive ion etching (RIE) of GaN epilayers while using nanoporous regimented free-standing alumina as a top etching mask [9]. As a key advantage, this top-down nanofabrication route, in short, a second order self-assembly (SOSA) of $\mathrm{GaN}$, enables a facile synthesis of ultradense, uniform array of nanopores without compromising the key electronic characteristics of $\mathrm{GaN}$. To assess the potential for 


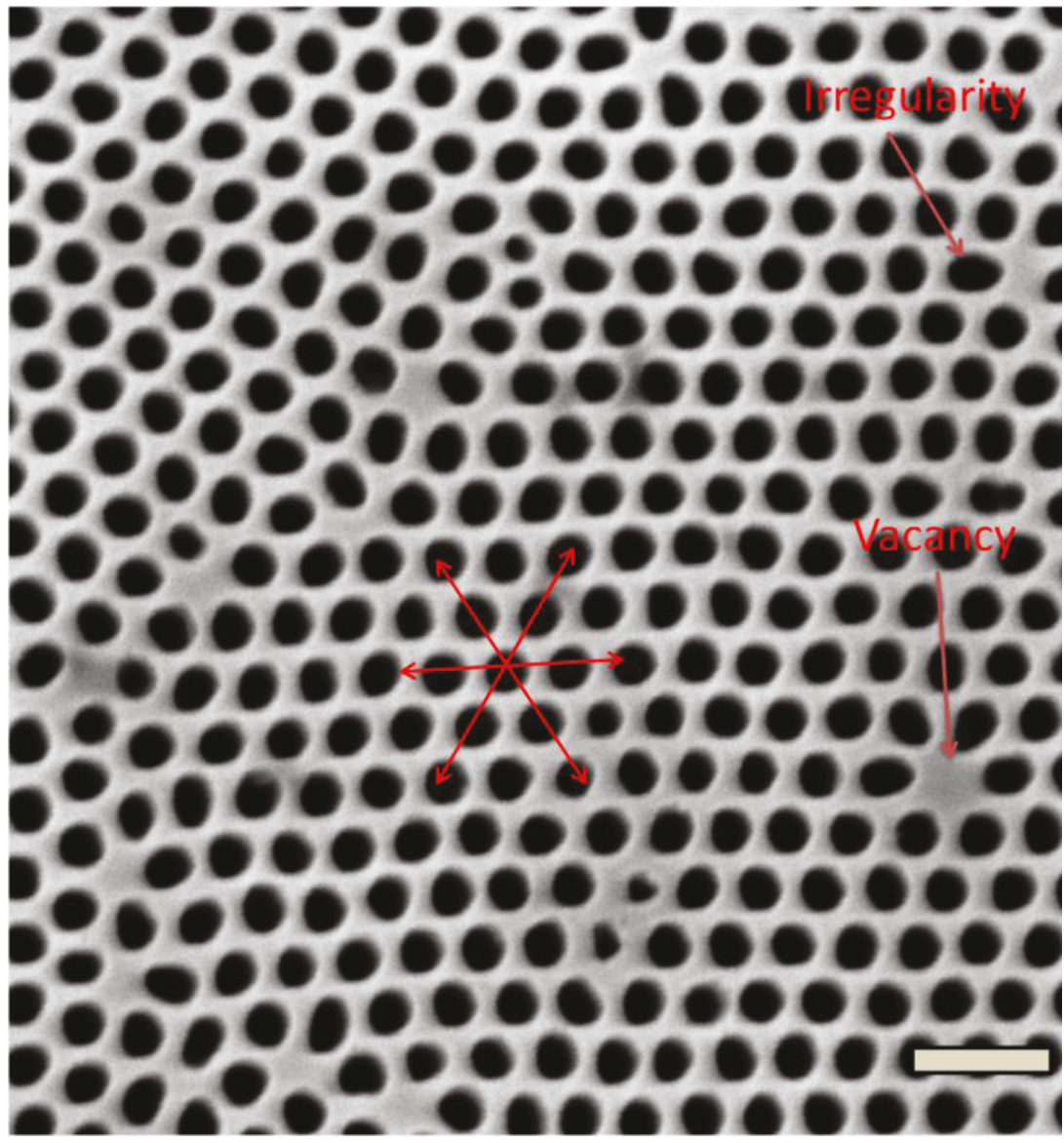

(a)

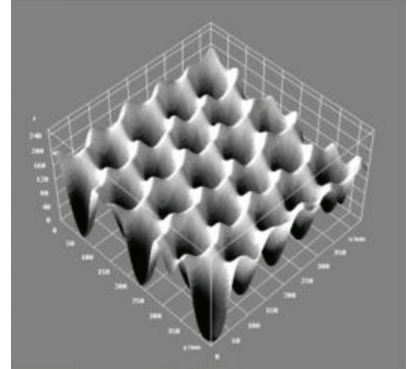

(b)

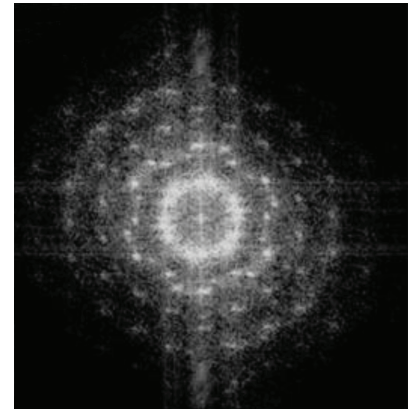

(c)

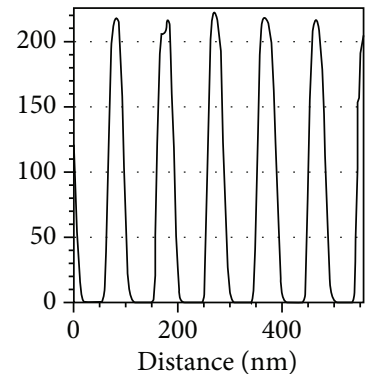

(d)

Figure 1: (a) Top view HRSEM image of np-GaN prepared by SOSA of GaN (bar is $200 \mathrm{~nm}$ ). The red lines represent nonpreferred light polarizations discussed in the text. (b) 3D/tilted-view HRSEM image. (c) Fast Fourier Transform of SEM image shown in (a) and (d) pore cross-sectional plot. The average pore diameter is $\sim 60 \mathrm{~nm}$, wall thickness is $\sim 33 \mathrm{~nm}$, and surface porosity is $\sim 42 \%$.

future device uses, in this study, we investigate the structural, strain and light emission characteristics of np-GaN produced by SOSA by performing a large-area scanning electron microscope imaging, Raman, optical reflection, temperature, and intensity-dependent photoluminescence spectroscopic characterizations, the details of which are presented and discussed below.

\section{Synthesis and Structural Properties of np-GaN}

Below, we briefly discuss the SOSA approach used to fabricate the samples. The SOSA technique is a variant of nonlithographic, high-throughput low-cost two-step surface nanopatterning technique which is used to produce ultrahigh dense, highly crystalline lateral superlattices of nanopores. It combines advantages of well-established electrochemical self-assembly and top-down plasma-based processing to produce ordered, high aspect ratio nanopores in elemental, compound, and alloyed semiconductors. As a first step, a free-standing nanoporous alumina template is produced by a two-step anodization approach [16]. To open up channels, a barrier layer at the bottom of the template is removed by a wet-etching. The resultant template is transferred on top of the semiconductor surface, which is next subject to dry etching. In general, by varying the anodization conditions, such as the electrolyte type, temperature, and current density, nanoporous masks with varying surface density and pore diameter can be readily produced.

In this work, free-standing alumina templates with an average pore diameter of $\sim 60 \mathrm{~nm}$ were fabricated and placed on top of epi-GaN grown by Molecular Beam Epitaxy (MBE) on top of c-Sapphire substrate. RIE was done using $\mathrm{Cl}_{2}$ (etchant) gas in a commercial-type plasma etch reactor using an optimized set of conditions, similar to those reported in [9]. The resultant np-GaN samples were transparent and mostly indistinguishable from as-grown epi-GaN to the eye. Figure 1(a) shows a top view SEM image of the resultant $\mathrm{np}-\mathrm{GaN}$. The average pore diameter and sidewall thickness are assessed to be $\sim 60$ and $33 \mathrm{~nm}$, respectively. The pore depth was limited to $\sim 300 \mathrm{~nm}$. The long-range order of the pores was further analyzed by carrying out a Fast Fourier Transform 


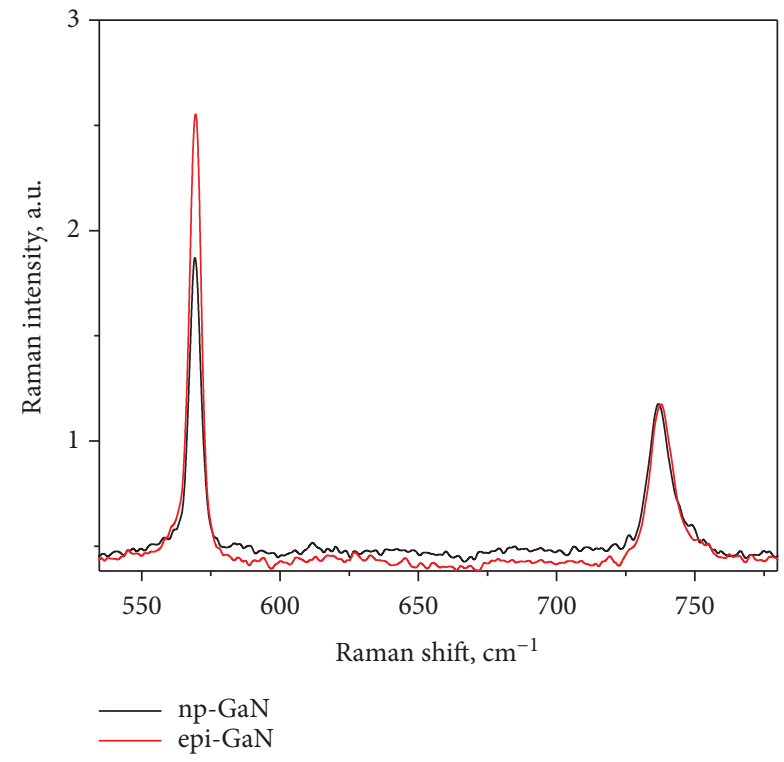

FIGURE 2: Raman spectra obtained in $z(x x) \bar{z}$ backscattering configuration on GaN nanopore arrays and epi-GaN.

(FFT) of the top view SEM image from Figure 1(a). The obtained FFT pattern, Figure 1(c), closely resembles a hexagon ring. This is consistent with the fact that the lateral superlattices are not totally free of morphological defects, which, according to Figure 1(a), include vacancies, shape irregularities, and grains.

\section{Raman and \\ Photoluminescence Spectroscopies}

Raman spectroscopy remains one of the key nondestructive characterization techniques ubiquitously used to probe phonon and plasmon elementary excitations in various materials. Apart from studying the basic aspects and properties of these excitations, the results can be also used to determine the crystal orientation, order, defects, strain, and carrier density in semiconductors, including GaN [17-19]. To probe the change in the strain characteristics as well as RIEinduced crystal damage (amorphization), micro-Raman spectroscopic measurements were performed comparatively on epi-GaN and np-GaN. The measurements were done using a Renishaw 2000 Raman spectrometer operating in the backscattering geometry. The spectra were excited with a $632 \mathrm{~nm}$ laser line, and the collected light was analyzed with a thermoelectrically cooled Si detector. The measurements were performed at the room temperature in $z(x x) \bar{z}$ geometry using Olympus Microscope equipped with 50x objective. According to Figure 2, the spectra are dominated by two peaks centered at $\sim 569 \mathrm{~cm}^{-1}$ and $736 \mathrm{~cm}^{-1}$ and attributed to $E_{2}$ and $A_{1}$ phonon modes, respectively, for both epi-GaN and $\mathrm{np}-\mathrm{GaN}$, in agreement with the Raman selection rules for wurtzite GaN.

$\mathrm{A} \sim 30 \%$ drop in the intensity of $\mathrm{E}_{2}$ peak is ascribed to a difference in the light coupling/scattering efficiency, and not to a Raman mode softening in np-GaN as a result of microstructural crystal damage. In fact, no peak broadening

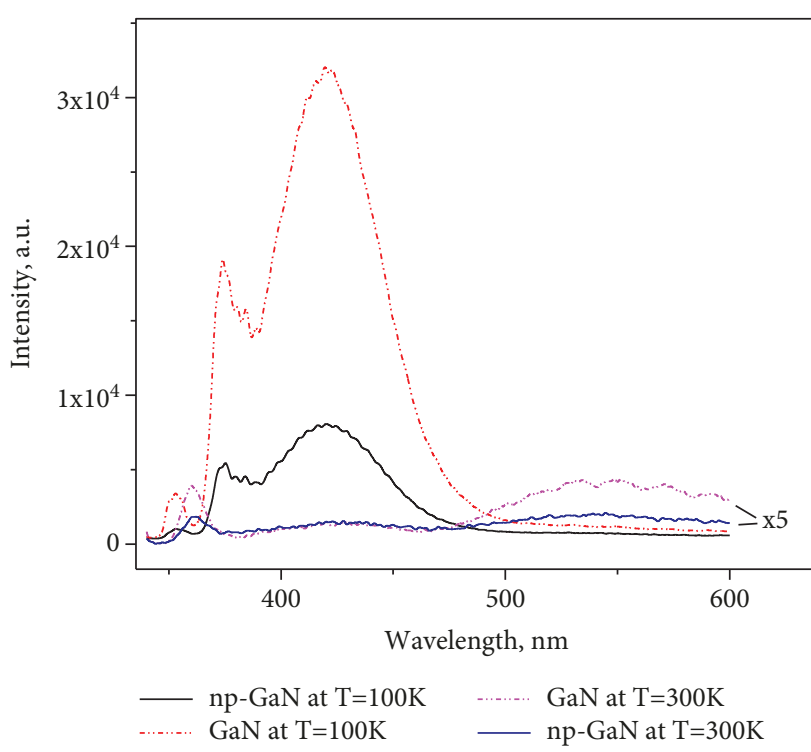

Figure 3: PL intensities of np-GaN (solid) vs. bulk epi-GaN (dot) obtained at $100 \mathrm{~K}$ and $300 \mathrm{~K}$.

for both phonon modes is confirmed for np-GaN, consistent with overall good crystal order of the processed sample. Yet, the peak position of $\mathrm{E}_{2}$ line exhibits a consistent red shift of $\sim 1.0 \mathrm{~cm}^{-1}$. This spectral shift is an indicator of a reduction of the compressive stress for $n p-G a N$. The amount of the stress relaxation can be assessed using the following equation:

$$
\Delta \omega=\alpha \sigma,
$$

where $\alpha$ is the proportionality constant and $\sigma$ stands for an in-plane biaxial stress. For $\mathrm{GaN}$, the proportionality constant is equal to $\sim 4.2 \mathrm{~cm}^{-1} \mathrm{GPa}^{-1}$, yielding the stress relaxation of $\sim 0.24 \mathrm{GPa}$.

Next, photoluminescence (PL) spectroscopy was carried out to investigate the band-edge and near-band-edge (NBE) optical emission characteristics of the structures. A Triax spectrometer ( $\mathrm{J}-\mathrm{Y}$ Horriba) equipped with two gratings mounted on computer-controlled turrets $(1200 \mathrm{gr} / \mathrm{mm})$ was used for light dispersions. A Hamasutsu photomultiplier tube (PMT) mounted on the exit port was used for visible range acquisitions. PL spectra were excited with the light produced by a $450 \mathrm{~W}$ Xenon lamp operating in conjunction with another Triax spectrometer. To improve lateral and vertical spatial resolutions, a two pin-hole confocal Olympus microscope equipped with $15 \mathrm{x}$ UV objective was used to collect intensity-dependent PL spectra.

Figure 3 shows both room and low temperature (300 and $100 \mathrm{~K}) \mathrm{PL}$ spectra of np-GaN and epi-GaN films by exciting samples with near-UV light $\left(325 \mathrm{~nm}, \sim 5 \mathrm{~mW} / \mathrm{cm}^{2}\right)$. At low $\mathrm{T}$, the PL emission from both samples is clearly dominated by one narrow and two broad emission bands at $\sim 353,374$, and $420 \mathrm{~nm}$, with the first and last bands attributed to excitonic and blue emission bands, respectively. The spacing between the first and second bands exceeds the GaN LO phonon energy of $\sim 92 \mathrm{meV}$, requiring us to rule out the possibility that this band is simply a phonon replica of the 
excitonic band. As its spectral position does not change with T, Figure 1S (Supplementary Materials), we assign this band to the transitions involving near-band-edge defect states. With the exciton binding energy of $\sim 23 \mathrm{meV}$ in $\mathrm{GaN}$ [20], the defect states located $\sim 194 \mathrm{meV}$ away from the band-edges are responsible for this emission and are tentatively attributed to $\mathrm{Ga}$ vacancies, a prevailing, acceptor-type native defect in GaN [21]. The assignment is in line with the results of the temperature-dependent intensity measurements discussed below.

Small PL ripples that appear at the long wavelength part of the spectra, Figure 3 (epi-GaN, $300 \mathrm{~K}$ ) are induced by light interference effects. For all temperatures, the net PL emission from np-GaN remains several times weaker compared to that from epi-GaN. As T increases and approaches $220 \mathrm{~K}$, the excitonic PL weakens by more than an order of magnitude, Figure S1 (Supplementary Materials), whereas the NBE PL band almost vanishes in both samples as a result of thermallyassisted depletion of the near-band-edge defect states.

Compared to bulk epi-GaN, np-GaN exhibits almost a threefold reduction in NBE emission levels, independent of the temperature. The intensity of the yellow defect emission band that only appears at $300 \mathrm{~K}$ remains almost fourfold weaker in the nanoporous sample.

A reduction of the refractive index contrast between air and nanoporous semiconductor leads to a drop in the light reflection losses at the air/semiconductor interface. According to Figure $2 S$ (Supplementary Materials), at the PL excitation wavelength of $320 \mathrm{~nm}$, the optical reflection coefficients are $\sim 14 \%$ in $\mathrm{np}-\mathrm{GaN}$ and $\sim 20 \%$ in epi-GaN. While a drop in the reflection was reported to result in stronger PL signals, this was not the case for the np-GaN templates studied by us. The reduction of the emission intensity, observed experimentally, is in part attributed to a decline in the electric field and, in turn, absorption strength for mesoscopic structures that feature a high aspect ratio and large dielectric constant mismatch [22]. In the case of np-GaN, the polarizations, for which light absorption is to be weaker, are shown by arrows in Figure 1(a). While morphological defects soften the polarization-absorption selection rules, the impact of these defects is likely to be very limited for our np-GaN samples owing to an overall good long-range order as verified by Fast Fourier Transform (FFT) results presented in Figure 1(c).

To probe the physical mechanisms regulating the optical emission processes in $\mathrm{np}-\mathrm{GaN}, \mathrm{T}$-dependent PL has been collected in the spectral range of $\sim 340-500 \mathrm{~nm}$. According to Figure 3, at $100 \mathrm{~K}$, the excitonic PL emission band exhibits a split into two excitonic bands with peaks centered at $\sim 350$ and $354 \mathrm{~nm}$ and ascribed to free and defect-bound excitons, respectively. At higher $\mathrm{T}$, both peaks exhibit a monotonic red shift and non-Arrhenius type intensity decline with $\mathrm{T}$ as it was closely fitted with $\sim \mathrm{T}^{-2}$ dependence, Figure $3 \mathrm{~S}$ (Supplementary Materials); likely as a result of an increase in the role of the phonon-exciton dissociations as $\mathrm{T}$ rises. A spectral shift of $\sim 30 \mathrm{meV}$ induced as $\mathrm{T}$ increases from 220 to $300 \mathrm{~K}$, Figure 4, the inset, is consistent with the reduction of the band gap of bulk GaN by $24 \mathrm{meV}$ [23].

Below, we further analyze the results of the roomtemperature excitation-dependent excitonic PL measurements.

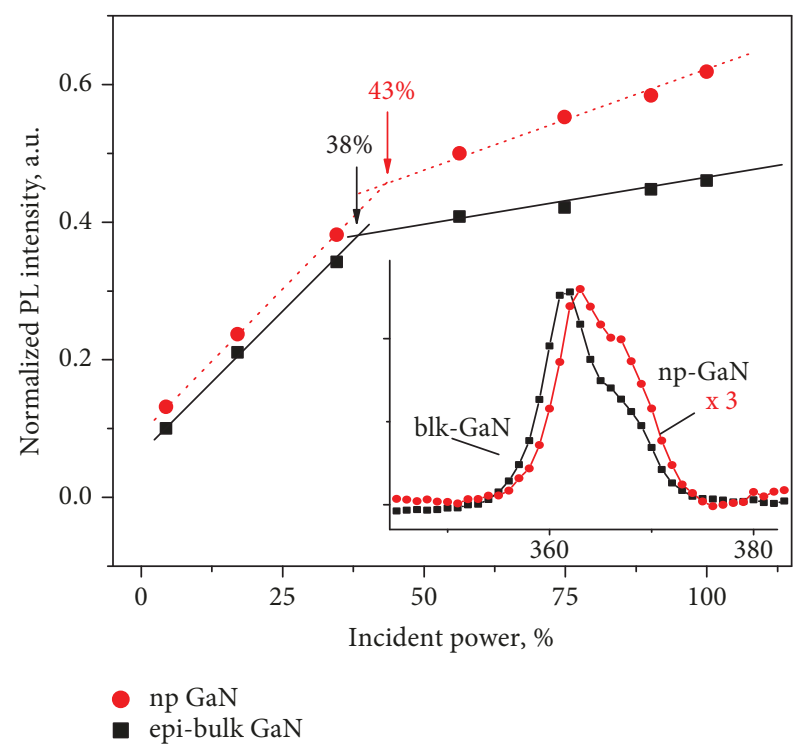

FIgURE 4: Room-temperature integral excitonic intensity vs. excitation intensity plots obtained for $\mathrm{np}-\mathrm{GaN}$ vs. bulk epi-GaN. The inset shows room temperature excitonic emission spectra obtained with a confocal microscope.

As seen in Figure 4, while the PL intensity increases linearly, it exhibits switching from one linear regime to another when excitation intensity reaches $\sim 40 \%$. We note that the switching thresholds were slightly different, i.e., 38\% for epi-GaN vs. $\sim 43 \%$ for $\mathrm{np}-\mathrm{GaN}$. Likewise, at the high-intensity regime, the excitonic emission from np-GaN is seen to exhibit a faster increase with the incident optical powers. The observed switching effect is next analyzed within the framework of the exciton recombination-generation dynamics, with the results presented and discussed below.

In the absence of any significant exciton diffusion, the following rate equation can be used to obtain the density of the free excitons, $n$ in both samples:

$$
\frac{d n}{d t}=G-\gamma n-\beta n^{2}
$$

where $G$ is the exciton generation rate, $\gamma$ is the free exciton decay rate coefficient, and $\beta$ is the nonlinear proportionality coefficient, with the last term accounting for biexciton dissociations. At the steady-state ( $c w$-illumination) and net low exciton densities (linear regime), the exciton density can be readily obtained: $n=G / \gamma$. Furthermore, $\gamma=\tau_{\text {ef }}^{-1}$, where $\tau_{\mathrm{ef}}$ stands for an effective lifetime of excitons. In the presence of two exciton loss channels, $\tau_{\text {ef }}$ can be obtained using $\tau_{\mathrm{ef}}^{-1}=\tau_{\mathrm{rad}}^{-1}+\tau_{\mathrm{nr}}^{-1}$, where $\tau_{\mathrm{rad}}$ and $\tau_{\mathrm{nr}}$ stand for radiative and nonradiative exciton lifetimes, respectively. Finally, the excitonic PL intensity, $I_{\text {exc }}$, can be readily obtained.

$$
I_{\text {exc }}=\frac{n}{\tau_{\mathrm{rad}}}=\frac{G}{\left(1+\tau_{\mathrm{rad}}\right) / \tau_{\mathrm{nr}}} .
$$


The above result correctly predicts a linear I vs. G dependence established experimentally. The result also implies that, under $c w$-illumination, switching between two linear emission regimes can be only invoked by an abrupt-like change in $\tau_{\mathrm{rad}} / \tau_{\mathrm{nr}}$. As reported prior [24], in the intermediate-to-high temperature range of $\sim 100-300 \mathrm{~K}$, $\tau_{\text {rad }}$ remains a monotonic function of $\mathrm{T}$. Therefore, the switching has to be attributed to a step-like decrease in $\tau_{\mathrm{nr}}$. While further investigations will be required, one of the possible explanations is a temperature-induced activation of the exciton-phonon nonradiative recombination channels when incident intensity approaches $\sim 40 \%$ levels. Due to a reduction in the light absorption strength, discussed earlier, a larger intensity threshold will be required for np-GaN, in agreement with the experimental data, Figure 4. A larger slope attained for np-GaN (high-intensity regime) for the excitation-emission curve (Figure 4) indicates a larger $\tau_{\mathrm{nr}}$ and, in turn, a more efficient radiative recombination of free excitons in $\mathrm{np}-\mathrm{GaN}$ as compared to epi-GaN.

\section{Summary}

In conclusion, structural and light emission characteristics of the regimented arrays of $\mathrm{np}-\mathrm{GaN}$ prepared by RIEbased SOSA technique have been investigated by performing Raman, optical reflection, and PL spectroscopies. The Raman results point to the compressive stress relaxation of $\sim 0.24 \mathrm{GPa}$ in $\mathrm{np}-\mathrm{GaN}$ as compared to as-grown bulk epi-GaN. According to the temperature-dependent PL measurements, the primary mechanism of the room-temperature PL emission is identified as excitonic and not interband free-carrier recombination. In contrast to prior reports, the net PL intensity is found to experience a near 3- to 4 -fold decrease in case of np-GaN, in part due to the polarization-absorption anisotropy. At the same time, as incident optical powers rise and exceed $\sim 43 \%$ threshold, the strength of excitonic PL is to undergo a much faster increase with the incident light intensity as a result of more efficient free exciton recombinations in $\mathrm{np}-\mathrm{GaN}$.

\section{Data Availability}

The data used to support the findings of this study are available from the corresponding author upon request.

\section{Conflicts of Interest}

The authors declare that there is no conflict of interest regarding the publication of this paper.

\section{Acknowledgments}

The work at UWM was supported by UWM RGI grant.

\section{Supplementary Materials}

Figure 1S presents $T$-dependent PL measurement data; the measurements were carried out in the temperature range of $100-300 \mathrm{~K}$ as discussed in the text. Figure 1S: PL intensities of np-GaN obtained at the temperature range of $100-300 \mathrm{~K}$. Optical reflection measurements were carried out using Ocean Optics SD2000 spectrometer in the spectral range of $\sim 215-800 \mathrm{~nm}$ on $\mathrm{np}-\mathrm{GaN}$ vs. epi-GaN thin films. According to Figure 2S, a reduction in the reflection for $\mathrm{np}-\mathrm{GaN}$ is evident up to $\sim 750 \mathrm{~nm}$. Figure $2 \mathrm{~S}$ : reflectance spectra of np-GaN and bulk epi-GaN. Log-log plot of excitonic peak intensity vs. $T$ data is shown in Figure $3 \mathrm{~S}$. The red line presents results of fitting the data with $I \sim T^{-2}$ function. Figure 3S: double-log plot of excitonic peak intensity of $\mathrm{np}-\mathrm{GaN}$ vs. $T$; the red line shows the results of fitting the data with $I \sim T^{-2}$ function. (Supplementary Materials)

\section{References}

[1] F. Schwierz, Solid State Electronics, vol. 49, no. 6, pp. 889-895, 2005.

[2] O. H. Nam, M. D. Bremser, T. S. Zheleva, and R. F. Davis, Applied Physics Letters, vol. 71, no. 18, pp. 2638-2640, 1997.

[3] Y.-F. Wu, B. P. Keller, P. Fini et al., IEEE Electron Device Letters, vol. 19, no. 2, pp. 50-53, 1998.

[4] Y.-F. Wu, A. Saxler, M. Moore et al., IEEE Electron Device Letters, vol. 25, no. 3, pp. 117-119, 2004.

[5] Y. Arakawa and A. Yariv, IEEE Journal of Quantum Electronics, vol. 22, no. 9, pp. 1887-1899, 1986.

[6] H. Jia, L. Guo, W. Wang, and H. Chen, Advanced Materials, vol. 21, no. 45, pp. 4641-4646, 2009.

[7] S.-H. Park, G. Yuan, D. Chen et al., Nano Letters, vol. 14, no. 8, pp. 4293-4298, 2014.

[8] W. S. Wong, T. Sands, N. W. Cheung et al., Applied Physics Letters, vol. 75, no. 10, pp. 1360-1362, 1999.

[9] N. A. Kouklin and J. Liang, "Ultradense GaN nanopillar and nanopore arrays by self-assembly nanopatterning," Journal of Electronic Materials, vol. 35, no. 5, pp. 1133-1137, 2006.

[10] C. B. Soh, C. B. Tay, R. J. N. Tan et al., Journal of Physics D: Applied Physics, vol. 46, no. 36, article 365102, 2013.

[11] K. J. Lee, S. J. Kim, J. J. Kim, K. Hwang, S. T. Kim, and S. J. Park, Optics Express, vol. 22, no. S4, p. A1164, 2014.

[12] J. Liang, S.-K. Hong, N. Kouklin, R. Beresford, and J. M. Xu, Applied Physics Letters, vol. 83, no. 9, pp. 1752-1754, 2003.

[13] A. P. Vajpeyi, S. Tripathy, S. J. Chua, and E. A. Fitzgerald, Physica E: Low-dimensional Systems and Nanostructures, vol. 28, no. 2, pp. 141-149, 2005.

[14] P. Pandey, M. Sui, M. Y. Li et al., Crystal Growth \& Design, vol. 16, no. 6, pp. 3334-3344, 2016.

[15] L. Zhang, S. Wang, Y. Shao et al., "One-step fabrication of porous GaN crystal membrane and its application in energy storage," Scientific Reports, vol. 7, no. 1, p. 44063, 2017.

[16] S. Bandyopadhyay, L. Menon, N. Kouklin, H. Zeng, and D. J. Sellmyer, Journal of Electronic Materials, vol. 28, no. 5, pp. 515-519, 1999.

[17] H. Siegle, P. Thurian, L. Eckey et al., Applied Physics Letters, vol. 68, no. 9, pp. 1265-1266, 1996.

[18] P. Perlin, C. Jauberthie-Carillon, J. P. Itie, A. San Miguel, I. Grzegory, and A. Polian, Physical Review B, vol. 45, no. 1, pp. 83-89, 1992.

[19] W. Rieger, T. Metzger, H. Angerer, R. Dimitrov, O. Ambacher, and M. Stutzmann, Applied Physics Letters, vol. 68, no. 7, pp. 970-972, 1996. 
[20] W. Shan, B. D. Little, A. J. Fischer et al., "Binding energy for the intrinsic excitons in wurtzite GaN," Physical Review B, vol. 54, no. 23, pp. 16369-16372, 1996.

[21] M. A. Reshchikov and H. Morkoç, "Luminescence properties of defects in GaN," Journal of Applied Physics, vol. 97, no. 6, article 061301, 2005.

[22] N. Kouklin, H. Chik, J. Liang et al., "Highly periodic, threedimensionally arranged InGaAsN: Sb quantum dot arrays fabricated nonlithographically for optical devices," Journal of Physics D: Applied Physics, vol. 36, no. 21, pp. 2634-2638, 2003.

[23] O. Lagerstedt and B. Monemar, Journal of Applied Physics, vol. 45, no. 5, pp. 2266-2272, 1974.

[24] O. Brandt, J. Ringling, K. H. Ploog, H. J. Wünsche, and F. Henneberger, Physical Review B, vol. 58, no. 24, pp. R15977-R15980, 1998. 


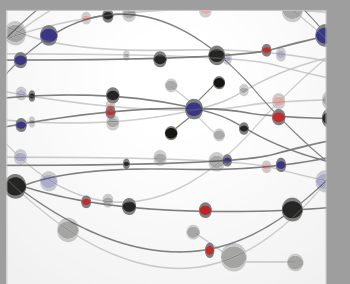

The Scientific World Journal
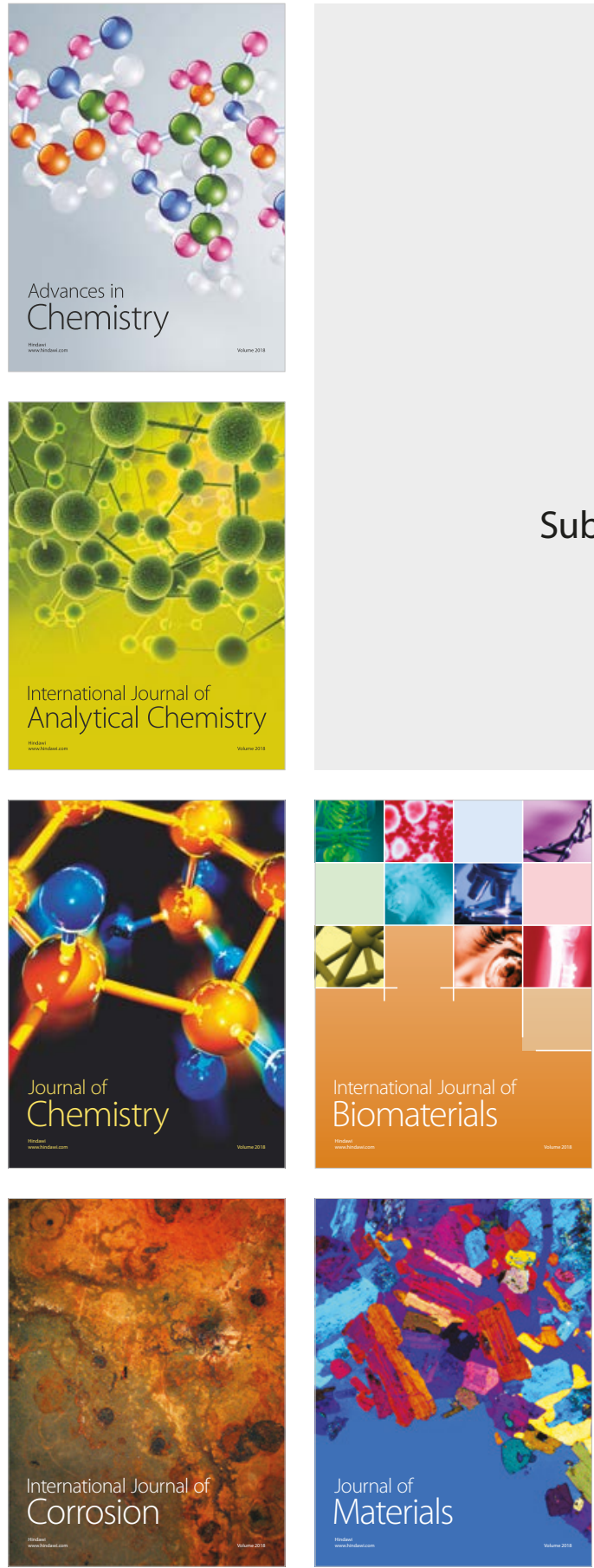

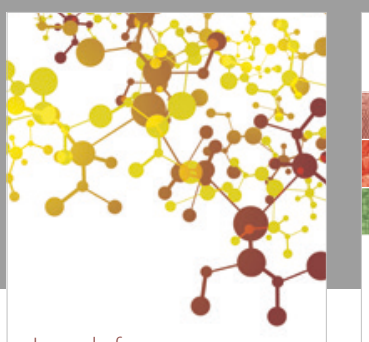

Journal of

Applied Chemistry
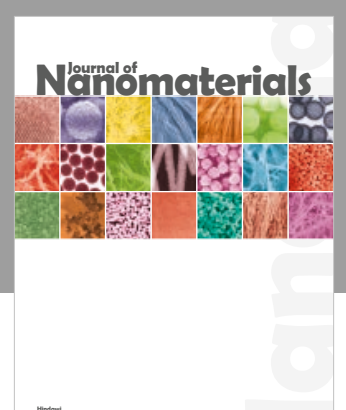



Scientifica



Polymer Science



Physical Chemistry
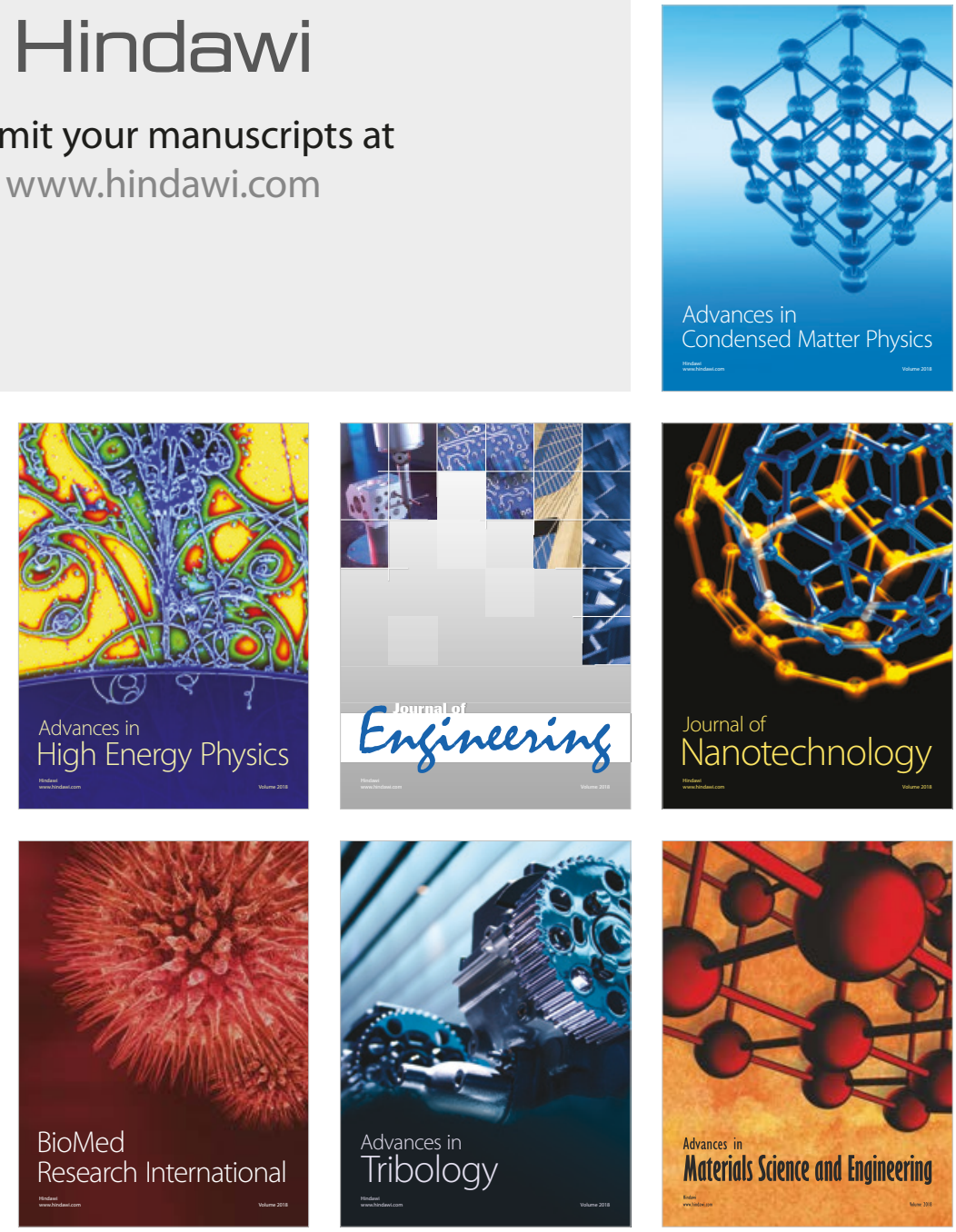